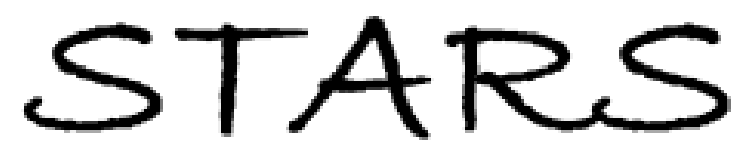

University of Central Florida

STARS

Faculty Bibliography 2010s

Faculty Bibliography

$1-1-2012$

\title{
Liquid-based infrared optical switch
}

Hongwen Ren

Su Xu

University of Central Florida

Yifan Liu

University of Central Florida

Shin-Tson Wu

University of Central Florida

Find similar works at: https://stars.library.ucf.edu/facultybib2010

University of Central Florida Libraries http://library.ucf.edu

This Article is brought to you for free and open access by the Faculty Bibliography at STARS. It has been accepted for inclusion in Faculty Bibliography 2010 s by an authorized administrator of STARS. For more information, please contactSTARS@ucf.edu.

\section{Recommended Citation}

Ren, Hongwen; Xu, Su; Liu, Yifan; and Wu, Shin-Tson, "Liquid-based infrared optical switch" (2012). Faculty Bibliography 2010s. 3183.

https://stars.library.ucf.edu/facultybib2010/3183

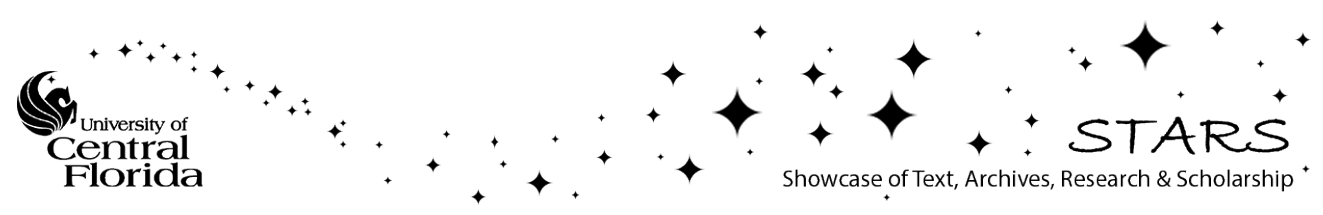




\section{Liquid-based infrared optical switch}

Cite as: Appl. Phys. Lett. 101, 041104 (2012); https://doi.org/10.1063/1.4738995

Submitted: 28 March 2012 . Accepted: 09 July 2012 . Published Online: 23 July 2012

Hongwen Ren, Su Xu, Yifan Liu, and Shin-Tson Wu

\section{ARTICLES YOU MAY BE INTERESTED IN}

A microfluidic $2 \times 2$ optical switch

Applied Physics Letters 85, 6119 (2004); https://doi.org/10.1063/1.1839281

Variable-focus liquid lens for miniature cameras

Applied Physics Letters 85, 1128 (2004); https://doi.org/10.1063/1.1779954

Tunable liquid microlens

Applied Physics Letters 82, 316 (2003); https://doi.org/10.1063/1.1536033

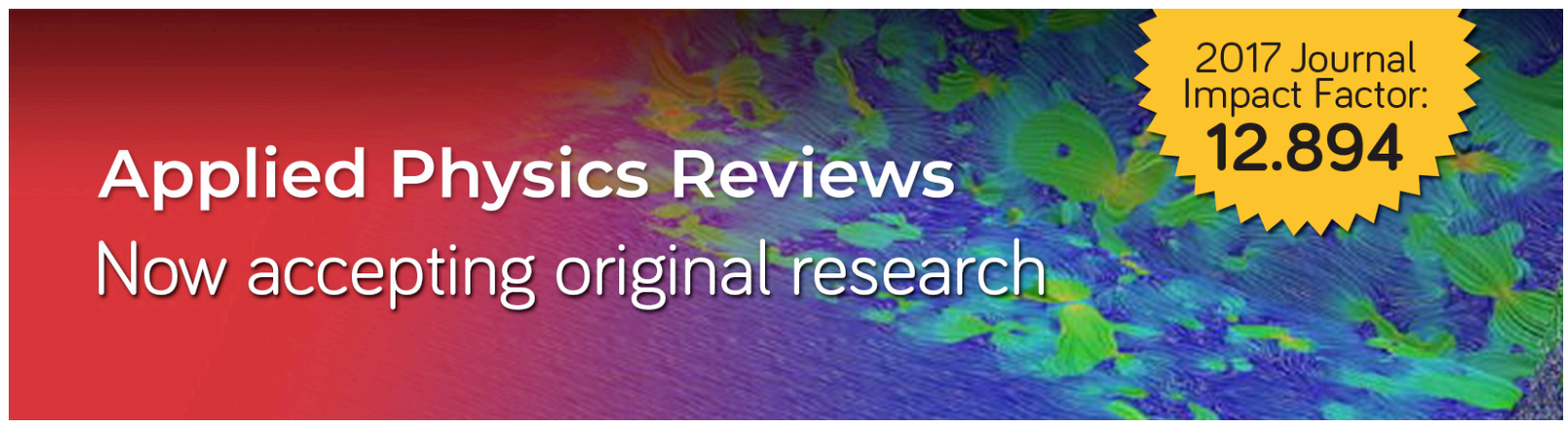




\title{
Liquid-based infrared optical switch
}

\author{
Hongwen Ren, ${ }^{1, a)} \mathrm{Su} \mathrm{Xu},{ }^{2}$ Yifan Liu, ${ }^{2}$ and Shin-Tson $\mathrm{Wu}^{2}$ \\ ${ }^{1}$ Department of Polymer Nano-Science and Technology, Chonbuk National University, Jeonju, \\ Chonbuk 561-756, Korea \\ ${ }^{2}$ CREOL, The College of Optics and Photonics, University of Central Florida, Orlando, Florida 32816, USA
}

(Received 28 March 2012; accepted 9 July 2012; published online 23 July 2012)

\begin{abstract}
We report an infrared (IR) optical switch using a position-shifting glycerol droplet. The droplet is surrounded by density-matched oil. In the voltage-on state, the droplet shifts in one direction. Upon removing the voltage, the droplet returns to its original position with the aid of interfacial tensions. Due to the strong absorption of glycerol at $1.55 \mu \mathrm{m}$, our IR optical switch shows $\sim 95: 1$ contrast ratio and $\sim 200 \mathrm{~ms}$ response time. Such a device is promising for fiber optical switch and various IR optical attenuators. (C) 2012 American Institute of Physics. [http://dx.doi.org/10.1063/1.4738995]
\end{abstract}

Fluidic optical switch is an attractive device for light shutter, variable optical attenuator, adaptive iris, and display. Various optical switches, such as polymer-dispersed liquid crystal (PDLC), ${ }^{1-4}$ microfluidic, ${ }^{5,6}$ electrowetting, ${ }^{7-11}$ and dielectrophoretic effect ${ }^{12-15}$ have been developed. However, most of these approaches are aimed for visible light and very few are suitable for infrared (IR) applications. ${ }^{16}$

PDLC, which controls the incident light by scattering, has been proposed for IR light switch. ${ }^{3}$ PDLC has some advantages, such as polarization independence, simple structure, and easy fabrication. But because of the forward scattering, its optical attenuation is very sensitive to the distance between the device and the detector. Microfluidic devices have also been demonstrated to modulate IR light. Liquid flows into microchannels under a pressure and IR light is switched by manipulating the liquid's position. However, such a device requires a mechanical driving system $^{5}$ or a complicated fabrication procedure ${ }^{6}$ or both. Electrowetting devices steer the IR light either by the total internal reflection from a mercury $\operatorname{slug}^{7}$ or by the deflection from a tunable liquid-liquid interface. ${ }^{11}$ With rapid development in optical communications, IR light switch has become a key component for regulating the optical power in an optical system. Therefore, there is an urgent need to develop a high performance IR light switch.

In this letter, we demonstrate an IR light switch with a glycerol droplet. In comparison to conventional liquids, glycerol has two unique merits: high absorption at $1.55 \mu \mathrm{m}$ and large dielectric constant. The former enables the glycerol droplet to evade or block the incident IR beam effectively, while the latter helps to lower the operation voltage. By using a 4.5-mm diameter glycerol droplet, we have demonstrated an IR light switch with $\sim 95: 1$ contract ratio and $\sim 200 \mathrm{~ms}$ response time.

Figure 1(a) shows the cross-sectional structure of the liquid cell at $\mathrm{V}=0$. Two immiscible liquids are sandwiched between glass substrates. One liquid $(\mathrm{L}-1)$ forms a droplet and the other liquid $(\mathrm{L}-2)$ fills the surrounding. The two liquids are density matched, thus the gravity effect during the droplet shifting is negligible when the cell is placed in

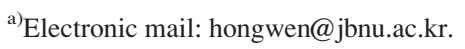

vertical direction. Moreover, L-2 helps to enhance the mechanical stability of the device, in case of shaking or vibrating. To actuate the droplet, the inner surface of the bottom glass substrate is coated with interdigitated electrodes (marked in red), followed by a thin polyimide (PI) layer (marked in green) and a thin teflon layer (marked in shades) sequentially. A hole, partially contacting with the edge of the striped electrodes, is patterned on the teflon layer, pinning down the droplet position at the relaxed state. Both the PI layer and teflon layer help to prevent the carrier injection from the electrodes, because of their high dielectric breakdown voltage and low leakage density. Due to the low surface tension of teflon, the droplet exhibits a minimal surface-to-volume ratio on the teflon surface.

For a stable droplet without any external force, the interfacial tensions satisfy following equation: ${ }^{17}$

$$
\gamma_{1}+\gamma_{3} \cos \theta=\gamma_{2}
$$

where $\gamma_{1}, \gamma_{2}$, and $\gamma_{3}$ denote the interfacial tension of $\mathrm{L}-1 /$ dielectric layer, $\mathrm{L}-2 /$ teflon, and $\mathrm{L}-1 / \mathrm{L}-2$, respectively, and $\theta$ is the contact angle of $\mathrm{L}-1$ on the teflon surface.

When a voltage is applied, the droplet experiences a dielectric force as ${ }^{18,19}$

$$
F=\frac{1}{2} \varepsilon_{0}\left(\varepsilon_{L-1}-\varepsilon_{L-2}\right) \nabla E^{2},
$$

where $\varepsilon_{0}, \varepsilon_{L-1}$, and $\varepsilon_{\mathrm{L}-2}$ represent the permittivity of free space, the dielectric constant of $\mathrm{L}-1$ and $\mathrm{L}-2$, respectively, and $\nabla E$ is the electric field gradient. Because of such a dielectric force, the system balance shown in Eq. (1) is broken. If $\varepsilon_{\mathrm{L}-1}>\varepsilon_{\mathrm{L}-2}$, the droplet would shift rightward to the high electric field intensity region, as Fig. 1(b) shows. When the whole droplet is pulled to the region with embedded electrodes, the motion will gradually stop because the left side and right side of the droplet bear an equal dielectric force but in opposite directions.

The motion of the droplet can be well described by the balance between dielectric force and interfacial tensions. If the droplet reaches a stable state as shown in Fig. 1(b), then along the indium-tin-oxide (ITO) stripe direction the system satisfies the following equation: 

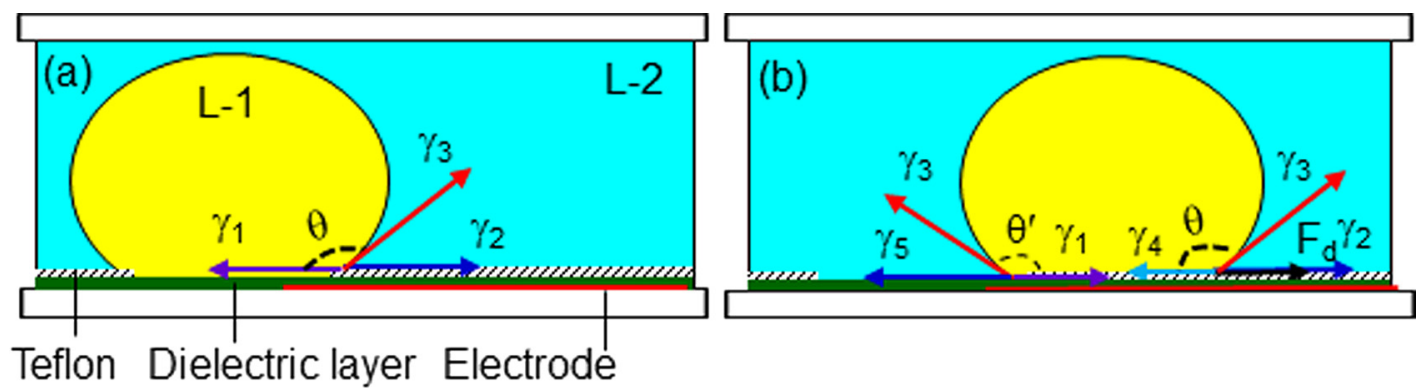

Teflon Dielectric layer Electrode

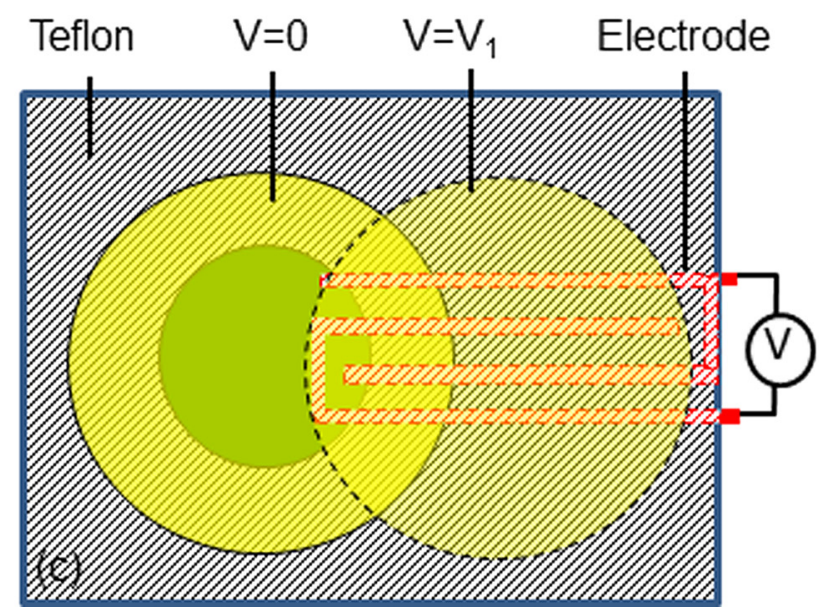

FIG. 1. Schematic structure of the cell and its operation mechanism. (a) The droplet pinned down on the hole surface at $V=0$, (b) the droplet moved to the right side at $\mathrm{V}=\mathrm{V}_{1}$, and (c) the top view showing the position of the droplet at $\mathrm{V}=0$ and $\mathrm{V}=\mathrm{V}_{1}$. The droplet size and electrode dimensions are not drawn by scale.

$$
\gamma_{1}+\gamma_{2}+\cos \theta^{\prime} \gamma_{3}+F_{d}=\gamma_{4}+\cos \theta \gamma_{3}+\gamma_{5}
$$

where $\gamma_{4}$ and $\gamma_{5}$ denote the interfacial tension of $\mathrm{L}-1 /$ teflon and $\mathrm{L}-2 /$ dielectric layer, respectively; $F_{d}$ is the horizontal component of dielectric force; and $\theta^{\prime}$ is the contact angle of $\mathrm{L}-1$ on the PI surface. From Eq. (3), as $F_{d}$ increases, $\gamma_{4}$ and $\theta$ will change accordingly before the whole droplet totally shifts to the region with embedded electrodes. If the surface tension of the droplet is very large, then the change of $\theta$ is rather limited. Instead, $\gamma_{4}$ will be the main varying factor because the droplet will go through a surface change from the dielectric layer surface to teflon when it shifts rightward. If $\theta \approx \theta^{\prime}$, then Eq. (3) is simplified to

$$
\gamma_{4}+\gamma_{5}=\gamma_{1}+\gamma_{2}+F_{d}
$$

When the voltage is removed $\left(F_{d}=0\right)$, the force balance in Eq. (4) is broken again. Since $\gamma_{4}+\gamma_{5}>\gamma_{1}+\gamma_{2}$, the droplet will be pulled back to its original position by interfacial tensions. To further illustrate the device structure, Fig. 1(c) depicts the top view of the device. Compared with the position at $\mathrm{V}=0$, the shifted droplet at $\mathrm{V}=\mathrm{V}_{1}$ covers a new area in addition to the overlapped region. The alternation between the original state $(\mathrm{V}=0)$ and the shifted state $\left(\mathrm{V}=\mathrm{V}_{1}\right)$ can be used for light switch.

To generate fringing fields, we adopt interdigitated ITO electrodes as Fig. 1(c) shows. The electric field distribution is simulated (Techwiz software) using Cartesian coordinate system. The $x$-axis is perpendicular to the stripe and $z$-axis is perpendicular to the glass surface. The ITO electrode width is $100 \mu \mathrm{m}$ and electrode gap is $20 \mu \mathrm{m}$. The distance along $z$-axis to the electrode surface is $50 \mu \mathrm{m}$. Figure 2 shows the simulated electric field intensity distribution at $\mathrm{V}=100$ $\mathrm{V}_{\text {rms. }}$. The electric field can be decomposed into two components: across the stripe $\mathrm{E}_{\mathrm{x}}$ (left) and along the cell gap direction $\mathrm{E}_{\mathrm{z}}$ (right). There is no electric field along the stripes ( $y$ axis). The electric field is the strongest at the electrode edges but decreases quickly as the distance departs from the edge. Therefore, the droplet border bears the strongest dielectric force along the ITO stripes.

In our experiments, two immiscible liquids glycerol $\left(\varepsilon_{1} \sim 47, \gamma_{\mathrm{g}} \sim 63\right.$ dyne $/ \mathrm{cm}, \rho_{\mathrm{g}}=1.25 \mathrm{~g} / \mathrm{cm}^{3}$, purity $\geq 99.5 \%$, from Sigma-Aldrich) and SantoLight ${ }^{\mathrm{TM}}$ Optical Fluids SL-5267 $\left(\varepsilon_{2} \sim 5, \gamma_{\mathrm{o}} \sim 40, \rho_{\mathrm{o}} \sim 1.26 \mathrm{~g} / \mathrm{cm}^{3}\right)$ are chosen as L-1 and $\mathrm{L}-2$, respectively. Since their densities match well, the gravity effect on shifting the droplet is minimized if the cell is vertically placed. Figure 3 shows the measured transmission spectra of the two liquids ( $\sim 4 \mathrm{~mm}$ thickness). At $\lambda=1.55 \mu \mathrm{m}, \mathrm{L}-1$ is opaque while $\mathrm{L}-2$ is transparent.

To prove concept, we prepared a liquid cell as depicted in Fig. 1(a). A glass plate with interdigitated ITO stripes was adopted as the bottom substrate. The electrode width and gap are $100 \mu \mathrm{m}$ and $20 \mu \mathrm{m}$; the same as those we used in simulation. The ITO surface was first spin-coated with a PI layer ( $\sim 1 \mu \mathrm{m}$ thickness) and post baked at $270{ }^{\circ} \mathrm{C}$, followed by a teflon layer ( $\sim 1 \mu \mathrm{m}$ thickness). We chose teflon AF solution grade 400S1-100-1 (from DuPont) because of its excellent hydrophobic and dielectric properties. ${ }^{20}$ After post-baking at $330{ }^{\circ} \mathrm{C}$ for $30 \mathrm{~min}$, the teflon layer was patterned with a 3.5-mm-diameter hole. The hole partially contacts with the electrode tips (Fig. 1(c)). The area covered by teflon exhibits a very low tension $(\gamma \sim 18 \mathrm{mN} / \mathrm{m})$ at the room temperature. 

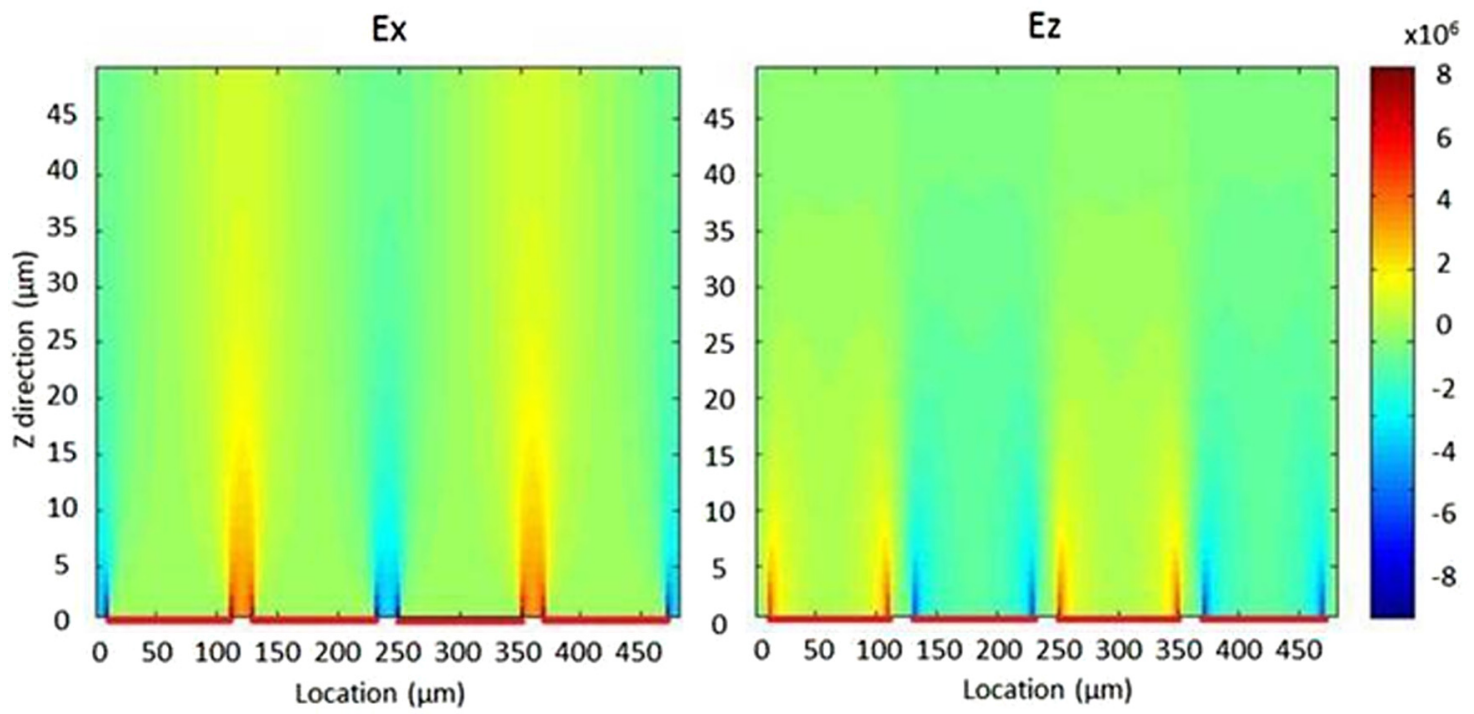

FIG. 2. Simulated electric field distribution: (a) along $x$-axis and (b) along z-axis. The ITO electrode width is $100 \mu \mathrm{m}$, electrode gap is $20 \mu \mathrm{m}$, and the distance along $\mathrm{z}$-axis to the electrode surface is $50 \mu \mathrm{m}$. The device structure is not drawn by scale.

While the surface tension of the PI surface (hole area) is relatively high $(\sim 40 \mathrm{mN} / \mathrm{m})$. After that, a small amount of glycerol was dispensed into the hole. Once the droplet border touched the teflon surface, it formed a spherical shape with a minimal surface-to-volume ratio. Then we filled the surrounding of the droplet with SL-5267 oil. The diameter of the droplet aperture (the touching area on the bottom glass plate) was controlled to be $\sim 4.5 \mathrm{~mm}$. Another glass plate was used as top substrate to cover the two liquids. The cell gap was controlled to be $\sim 5 \mathrm{~mm}$ using glass spacers, and the periphery of the cell was hermetically sealed by adhesive glue. Such a distance is safe to prevent the dome of the droplet from touching the top glass plate.

To study the electro-optical properties of the droplet, we observed the cell using an optical microscope. Because the droplet size is fairly large, the microscope cannot focus well on the entire droplet. At $\mathrm{V}=0$, when focusing on the dome area of the droplet we observed a large bright spot (dome) surrounded by a black border (Fig. 4(a)). As the voltage is gradually increased to $35 \mathrm{~V}_{\mathrm{rms}}$, the bright spot begins to shift along the ITO stripes, implying that the whole droplet shifts along ITO stripes. At $45 \mathrm{~V}_{\text {rms }}$, the shifting of the droplet becomes more noticeable (Fig. 4(b)). When the voltage is

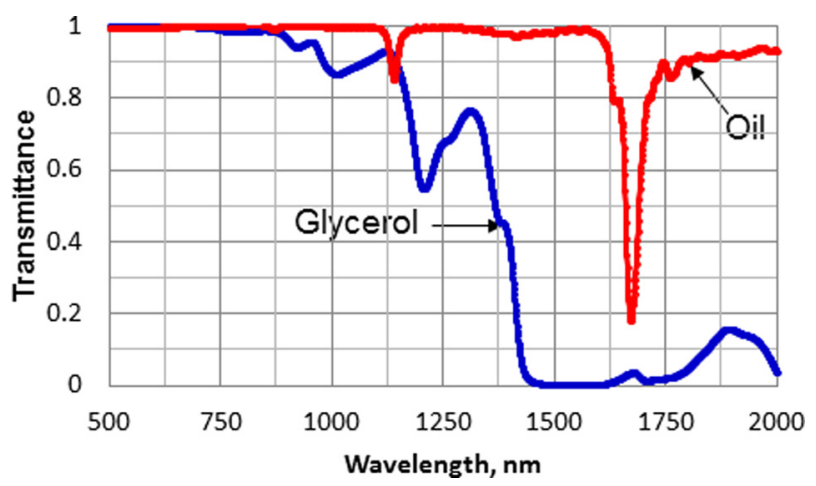

FIG. 3. Measured transmission spectra of glycerol and optical oil (SL-5267) from $500 \mathrm{~nm}$ to $2000 \mathrm{~nm}$. The thickness of each liquid is $\sim 4 \mathrm{~mm}$. increased to $60 \mathrm{~V}_{\text {rms }}$, the droplet shifts dramatically (Fig. 4(c)). Upon removing the voltage, the droplet returns to its original state. To visually observe the dynamic shifting of the droplet (between 0 and $100 \mathrm{~V}_{\mathrm{rms}}$ ), a multimedia of Fig. 4(a) showing the movement of the droplet is given as well (enhanced online). At $\mathrm{V}=100 \mathrm{~V}_{\mathrm{rms}}$, the droplet's dome (the bright spot) has already shifted out of the visual field.

According to the simulation results shown in Fig. 2, at $\mathrm{V}=100 \mathrm{~V}_{\mathrm{rms}}$, the peak electric field reaches as high as $8 \mathrm{~V}_{\mathrm{rms}} / \mu \mathrm{m}$ at the ITO edges. Nevertheless, such strong field only distributes in the PI layer, whereas the field exerted on the glycerol droplet is relatively weak. The glycerol has a purity of $>99.5 \%$ and the ionic impurity moving into the surrounding oil can be neglected. Besides, both PI layer and teflon layer help to prevent the carrier injection into the cell. The breakdown field strength of a typical teflon layer is $\sim 600-1730 \mathrm{kV} / \mathrm{cm}$, and that of a typical PI layer is $\sim 1.3-3.3$ $\mathrm{MV} / \mathrm{cm}^{21-23}$ Last but not least, AC rather than DC voltage operation also helps to suppress the charge effect. Therefore, even driven at $100 \mathrm{~V}_{\text {rms }}$ our device reproduces the droplet movement very well, as shown in the video.

Next, we evaluated the droplet's performance as an IR light switch at $1.55 \mu \mathrm{m}$. Figure 5(a) depicts a simple setup for characterizing the electro-optical properties. The cell was placed in vertical position. A laser beam (LAS DFB-1550-6, $\lambda=1.55 \mu \mathrm{m}$, Laser Max) was normally incident upon the ITO electrode area and close to the border of the droplet at $\mathrm{V}=0$. The diameter of the laser beam was controlled to be $\sim 4 \mathrm{~mm}$ by an iris diaphragm, slightly smaller than that of the glycerol droplet. The transmitted light intensity was detected by a photodiode. At $\mathrm{V}=0$ (the left), the laser beam passed through the region filled with SL-5267, and the transmitted intensity was the highest. At $\mathrm{V}=\mathrm{V}_{1}$ (the right), the droplet shifted upward and blocked the laser beam. Figure 5(b) shows the transmitted light intensity at various voltages. At $\mathrm{V}=35 \mathrm{~V}_{\mathrm{rms}}$, the light intensity starts to decrease, implying that the droplet begins to block the laser beam. At $\mathrm{V}=100 \mathrm{~V}_{\text {rms }}$, the light intensity is significantly decreased because the droplet almost blocks the 

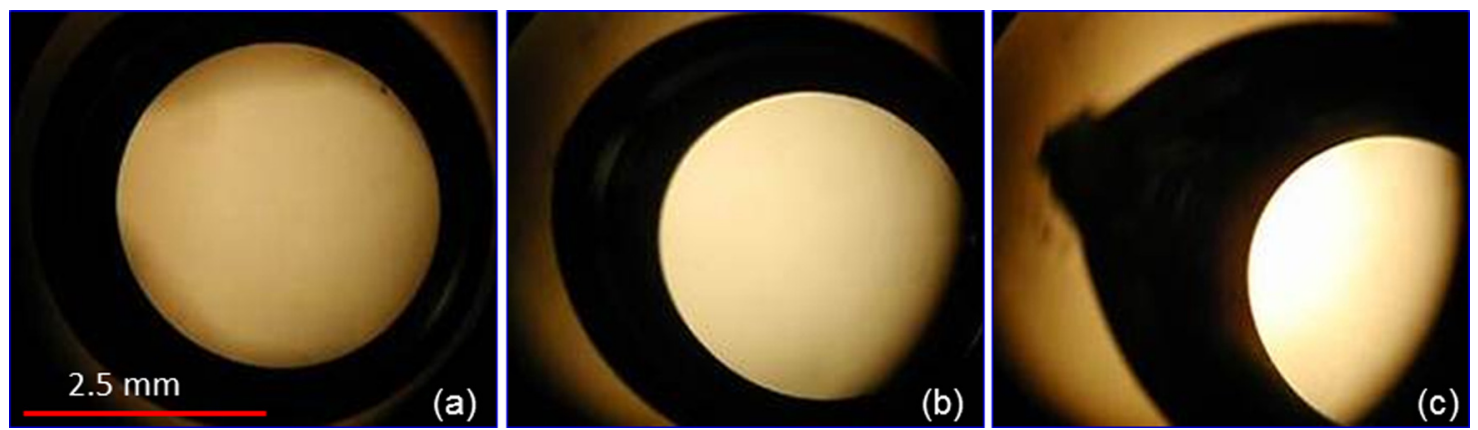

FIG. 4. The position change of glycerol droplet at different voltages: (a) $\mathrm{V}=0$, (b) $\mathrm{V}=45 \mathrm{~V}_{\mathrm{rms}}$, and (c) $\mathrm{V}=60 \mathrm{~V}_{\mathrm{rms}}$. The diameter of the droplet is $\sim 4.5 \mathrm{~mm}$. A dynamic multimedia of (a) is given as well (enhanced online) [URL: http://dx.doi.org/10.1063/1.4738995.1].

incident laser beam. The measured contrast ratio exceeds 95:1. The contrast ratio would be improved dramatically if the droplet could fully block the incident beam.

Response time is an important parameter for an optical switch. To measure response time, we applied a square voltage pulse with $100 \mathrm{~V}_{\mathrm{rms}}$ amplitude to the cell and recorded the time-dependent light transmission by a digital oscilloscope. Results are shown in Fig. 5(c). The cycle driving with two periods shows that the dynamic switching of the droplet repeats very well. It takes $\sim 80 \mathrm{~ms}$ for the droplet to largely block the laser beam and $\sim 200 \mathrm{~ms}$ to return to its original place.

Although the glycerol droplet switches the $1.55-\mu \mathrm{m}$ laser beam by absorption, the heating effect is negligible
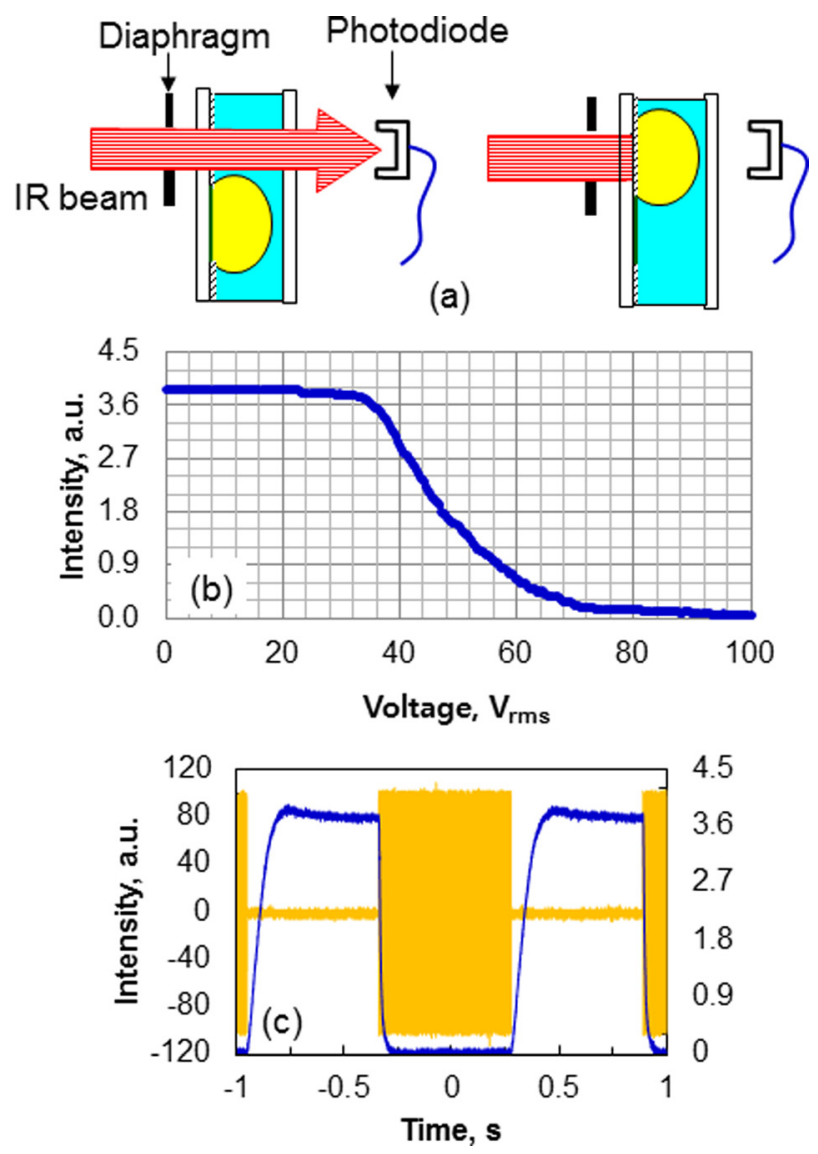

FIG. 5. Electro-optical characteristics of the IR switch at $\lambda=1.55 \mu \mathrm{m}$. (a) A simple setup, (b) intensity change vs. voltage, and (c) intensity change vs. time. because the laser power is weak $(\sim 4.5 \mathrm{~mW})$. Moreover, the generated heat would be dissipated by the surrounding oil and the glass. Therefore, thermal effect is not a concern in our device. The power consumption of the device is less than $\sim 1 \mathrm{~mW}$ under $1.5 \mathrm{kHz}$. The electro-optical effect of our cell is not charge-induced but fringe-field generated. To verify this assumption, we tested the droplet cell as a complex impedance $Z_{\text {load }}=|Z| e^{-i \varphi}$ (assuming no inductor). Here, the phase $\varphi$ of $Z_{\text {load }}$ encodes the phase relation between voltage $V_{0} \cos \omega t$ and current $I_{0} \cos (\omega t+\varphi)$. From our measurement (using Hioki 35632-50 LCR HiTester), we obtained $\varphi \sim 0.992 \cdot(\pi / 2)$ at $1.5 \mathrm{kHz}$, which agrees with the theoretical value of $\pi / 2$ very well. This indicates that the cell represents essentially a capacitive load and the Ohmic charge transport is negligible.

From Fig. 1(a), the droplet's aperture can be easily scaled up for different applications. Since the generated fringing field is independent of the cell gap, the operating voltage is insensitive to the droplet size. Although the employed ITO electrode gap is relatively wide $(20 \mu \mathrm{m})$, the droplet still shifts very well. To lower the operating voltage while keeping the desired electric field distribution, we could decrease the electrode gap to $5-10 \mu \mathrm{m}$ as used in display devices.

In our device, the two liquids have almost the same density and the droplet is firmly anchored to the hole surface at $\mathrm{V}=0$. As a result, even if the cell is placed in vertical direction, the droplet shape will not be deformed by the gravity effect. In the actuated state, the droplet experiences both dielectric force and interfacial tensions which assures good mechanical stability against shocks and vibrations. Although our device works very well at $1.55 \mu \mathrm{m}$, this device concept can be extended to mid-wavelength IR (Ref. 24) and longwavelength IR (Ref. 25) by choosing proper liquids in the desired spectral region. Different from a liquid lens, ${ }^{26-28}$ our device no longer depends on the refractive index difference, droplet profile, and shape distortion. Therefore, its electrooptical performances, such as response time, can be easily improved by only considering a few criteria, e.g., viscosity and interfacial tensions. Compared to other fluidic devices, our IR switch can also work in reflective mode if the inner surface of the top glass substrate is coated with a reflector. Therefore, wide spread application of this device concept in various optical communication systems is foreseeable.

In conclusion, we have demonstrated a simple IR light switch with a glycerol droplet actuated by a fringing field. 
For a 4.5-mm-diameter droplet, the measured contrast ratio is over 95:1 in transmissive mode and recovering time is $\sim 200 \mathrm{~ms}$ at $100 \mathrm{~V}_{\mathrm{rms}}$. The glycerol droplet is surrounded by density-matching oil, therefore the gravity effect is minimized and the device is mechanically stable against shocks and vibrations. Reflective mode can also be configured by coating a reflective material on the inner surface of the top glass substrate. Our device has advantages in scalable switching area, good optical performance, and good mechanical stability. Potential applications for optical fiber switch, IR shutter, attenuators, and filters are foreseeable.

H. Ren is indebted to the support from National Research Foundation of Korea under Basic Science Research Program 2010-0021680. The UCF group is partially supported by AFOSR under Contract No. FA95550-09-1-0170.

${ }^{1}$ P. S. Drzaic, J. Appl. Phys. 60, 2142 (1986).

${ }^{2}$ K. Takizawa, K. Kodama, and K. Kishi, Appl. Opt. 37, 3181 (1998).

${ }^{3}$ K. M. Chen, H. Ren, and S. T. Wu, Opt. Commun. 282, 4374 (2009).

${ }^{4}$ H. Ren, H. Xianyu, and S. T. Wu, J. Phys. D: Appl. Phys. 43, 365103 (2010).

${ }^{5}$ K. Campbell, A. Groisman, U. Levy, L. Pang, S. Mookherjea, D. Psaltis, and Y. Fainman, Appl. Phys. Lett. 85, 6119 (2004).

${ }^{6}$ Y. Zuta, I. Goykhman, B. Desiatov, and U. Levy, Opt. Express 18, 24762 (2010).

${ }^{7}$ J. Jacke, S. Hackwood, and G. Beni, Appl. Phys. Lett. 40, 4 (1982).

${ }^{8}$ R. A. Hayes and B. J. Feenstra, Nature 425, 383 (2003).
${ }^{9}$ Y. H. Lin, J. K. Li, T. Y. Chu, and H. K. Hsu, Opt. Express 18, 10104 (2010).

${ }^{10}$ C. U. Murade, J. M. Oh, D. van den Ende, and F. Mugele, Opt. Express 19, 15525 (2011).

${ }^{11}$ S. A. Reza and N. A. Riza, Opt. Commun. 282, 1298 (2009).

${ }^{12}$ C. G. Tsai and J. A. Yeh, Opt. Lett. 35, 2484 (2010).

${ }^{13}$ H. Ren and S. T. Wu, Opt. Lett. 35, 3826 (2010).

${ }^{14}$ H. Ren, S. Xu, D. Ren, and S. T. Wu, Opt. Express 19, 1985 (2011).

${ }^{15}$ H. Ren, S. Xu, and S. T. Wu, Lab Chip 11, 3426 (2011).

${ }^{16}$ S. T. Wu, Phys. Rev. A 33, 1270 (1986).

${ }^{17}$ A. W. Adamson and A. P. Gast, Physical Chemistry of Surfaces, 5 th ed. (Wiley, New York, 1990).

${ }^{18} \mathrm{P}$. Penfield and H. A. Haus, Electrodynamics of Moving Media (MIT, Cambridge, 1967).

${ }^{19}$ H. A. Pohl, Dielectrophoresis (Cambridge University Press, New York, 1978).

${ }^{20}$ G. G. Hougham, P. E. Cassidy, K. Johns, and T. Davidson, Fluoropolymers 2: Properties, (Plenum, New York, 1999).

${ }^{21}$ S. Diaham, S. Zelmat, M. L. Locatelli, S. Dinculescu, M. Decup and T. Lebey, IEEE Trans. Dielectr. Electr. Insul. 17, 18 (2010).

${ }^{22}$ R. Jensen, J. Cummings, and H. Vora, IEEE Trans. Compon., Hybrids, Manuf. Technol. 7, 384 (1984).

${ }^{23}$ H. Deligöz, T. Yalcinyuva, S. Özgümüs, and S. Yildirim, J. Appl. Polym. Sci.100, 810 (2006).

${ }^{24}$ Y. Chen, H. Xianyu, J. Sun, P. Kula, R. Dabrowski, S. Tripathi, R. J. Twieg, and S. T. Wu, Opt. Express 19, 10843 (2011).

${ }^{25}$ S. T. Wu, J. D. Margerum, H. B. Meng, C. S. Hsu, and L. R. Dalton, Appl. Phys. Lett. 64, 1204 (1994).

${ }^{26}$ S. Kuiper and H. W. Hendriks, Appl. Phys. Lett. 85, 1128 (2004).

${ }^{27}$ C. C. Chen, C. A. Chang, and J. A. Yeh, Opt. Express 14, 4101 (2006).

${ }^{28}$ H. Ren, H. Xianyu, S. Xu, and S. T. Wu, Opt. Express 16, 14954 (2008). 